\title{
Multimedia Document Architecture for Medical Applications
}

\author{
A. Karmouch and N.D. Georganas
}

\begin{abstract}
Document architecture is a fundamental element in the design architecture of picture archiving communication systems (PACS). This article gives an overview of a multimedia document architecture in terms of logical and layout structures and describes a method for organizing and modeling multimedia diagnostic reports. The proposed document model is based on the office document architecture (ODA) ISO standard with additional enhancements to satisfy the functionality required of future interworking PACS.
\end{abstract}

(C) 1989 by W.B. Saunders Company.

$\mathbf{T}$

HE USE of picture archiving and communication systems (PACS) is increasing because of the technological development of both medical imaging equipment (eg, computed tomography scanner, magnetic resonance imaging) and optical storage technologies. The widespread use of PACS will generate large amounts of information that must be organized and manipulated in a uniform way. Therefore, large capacity databases affording optimum management of multimedia data and offering high speed access to the stored information especially for images will be needed. There are two essential points to be considered in designing a multimedia database system: the document model and the database model. The document model defines the way information is organized while the database model defines the way this information is represented, described, and manipulated in the database. In this article we focus on the document model suitable for the medical environment, particularly for radiological applications. The document model is based on concepts of the Office Document Architecture (ODA) ${ }^{1}$ standard, which has been developed for the office environment by the ISO standard committee. Our intention is to adapt an existing standard on document architecture, such as ODA, rather than develop a new model from scratch. This approach has many advantages. The use of standards will facilitate the information exchange between future heterogeneous systems allowing intercommunications between geographically distributed hospitals. Also, the ability and the opportunity to contribute to the extension of the standard, in order to cover other needs and domains, will produce additional side benefits. Furthermore, the use of ODA will contribute to the understanding of the frontiers between the office and medical environment, which will reflect the specificities of the latter as compared with the general office.

Many PACS software systems are under development or are in a product development stage. ${ }^{2,3}$ It is unfortunate that none of them has addressed the document model paradigms that are a fundamental component of the PACS design and architecture. The reasons are mainly that the PACS equipment used to capture and to render images is not, in terms of technological evolution, mature enough to replace the traditional radiologist work station and to provide an image quality equivalent to that of $x$-ray films. A lot of work has to be done in this area, which constitutes the vital organs of PACS because of their fundamental role in the acquisition and display process of the information (digitized radiographs, voice dictation, image display, etc). Furthermore, the concept of multimedia documents is new and information in PACS is considered, by most of the existing systems, to be exclusively composed of digitized radiographs.

In this article, we give an overview of the document concept and justify the need for a common document model. Then we highlight the fundamental concepts of the multimedia document architecture and their use in the diagnostic reports illustrated by some examples. Finally, we give a conclusion with a brief discussion.

\section{THE DOCUMENT CONCEPT}

The document concept is used in the general office to communicate information between office workers. Information expressed in the document

From the Telecommunications Research Institute of Ontario, University of Ottawa Medical Communications Centre, Canada.

Supported by the Telecommunications Research Institute of Ontario (TRIO), Bell Canada, and Bell-Northern Research (BNR).

Address reprint requests to $A$. Karmouch, PhD, Telecommunications Research Institute of Ontario, University of Ottawa Medical Communications Centre, 161 Louis Pasteur, Ottawa, Ontario K1N 6N5, Canada.

(๑) 1989 by W.B. Saunders Company.

0897-1889/89/0202-0008\$03.00/0 
may be presented in different forms such as text, graphics, and images. To be read efficiently, the information must be organized according to a well-recognized logical and layout structure. Many organizations have some internal standards for structuring information on a paper document (eg, written medical reports), which may vary according to the document class, such as report, letter, patient forms, etc. Once created, documents are distributed or made available for consultation purposes. To read a document one would use the logical structure combined with the layout structure to efficiently understand the semantic intent of the document. Document organization is therefore an important concept that must be well defined in order to help the reader to understand the ideas expressed in the document.

In the electronic environment the document concept is a key factor in designing an integrated and distributed information system such as PACS. In such a system different tools (ie, applications) will be offered allowing the document to be created, manipulated, distributed, stored, retrieved, browsed, and annotated. A document will become the central object exchanged and manipulated by these tools (eg, editor, mailing, conference, database, hypermedia, etc) upon the request of the user, each application acting on the document according to the service it provides. For instance, the editor allows the radiologist to generate a diagnostic report while the database system provides functions for filing and retrieving reports together with the corresponding images. In this example, the editor and the database applications should have the same conceptual view (ie, data structure) of the diagnostic report in order to exchange its content, by means of a communication medium, in a homogeneous and uniform way without any conversion functions. Therefore, a common model describing the way the document must be organized and structured to be interchanged and processed by different applications is needed. The model is also useful for the users; it describes a common method that they can refer to in order to organize information in a document so that the readers of that document can easily "navigate" inside the document and browse its content.

\section{DOCUMENT ARCHITECTURE PRINCIPLES}

The types of documents that may be represented in the ODA document model are those frequently used in general office work such as reports, letters, forms, invoices, memoranda, etc. In practice such office documents often contain text, graphics, pictures and, in some cases, all of these within a single multimedia document. The purpose of the document model is to provide a method of describing the electronic representation of these kind of documents, including the type of information found in their content. The structured description of such documents is termed "architecture" of the document. A document architecture can be subdivided in three categories (Fig 1): logical structure; layout structure; and document content.

The logical structure provides a method for organizing the content of the document and it is intended to closely correspond to those aspects of the document structure related to the functional semantic of the document. The layout structure provides a method for organizing the content of the document into pages and areas within pages. The content is the information that we normally think a document includes (eg, sequence of paragraphs within a section or a chapter). Figure 1 illustrates the logical and layout structures of a document in terms of views.

\section{Logical Structure}

The logical structure provides a means of modeling the structure of a document in terms of logical objects that have meanings significant to the user. For example, the logical structure can be used to model a document in terms of chapters, sections, paragraphs, appendices, headings, footnotes, figures, etc. However, the logical structure model must be general enough to allow other kinds (eg, diagnostic report) of documents to be

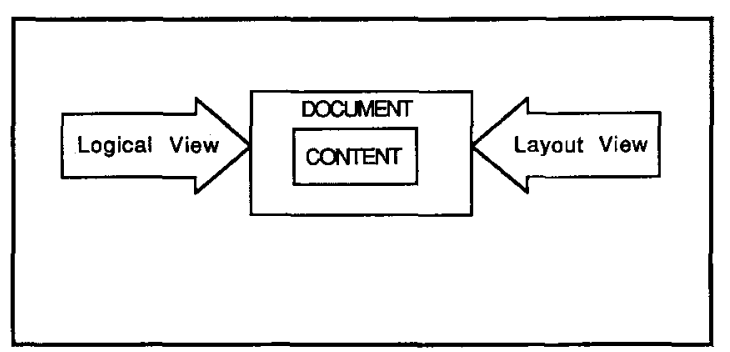

Fig 1. Logical and layout views of a document. 
defined in terms of logical objects and should not be restricted to a given kind of document. In addition, the model provides the means by which any such construction can be defined in terms of logical objects to be interpreted by the application user such as chapters, sections, paragraphs, footnotes, etc. In other words, the logical structure model is not dedicated to a particular kind of document but it allows any number of different kinds of documents to be represented in terms of logical objects so as to meet a wide variety of application needs. A logical structure of a document can include the following object types: document logical root (document as an entity); composite logical object (may represent a chapter, section, etc); and basic logical object (object to which a content portion is associated). An example of a logical structure of a diagnostic report is given in Figs 2 and 3.

\section{Layout Structure}

The layout structure provides a view of the content and is used to make explicit in the document those aspects concerning its position and other imaging properties on the presentation medium (screen or page). The layout structure describes how the content is to be allocated to pages and areas within pages.

The components available to describe the allocation of content are hierarchically related and are: (1) groups of related pages, termed page sets, eg, the pages which contain a particular chapter or section of a document; (2) pages that are used for relating positioning of the content to

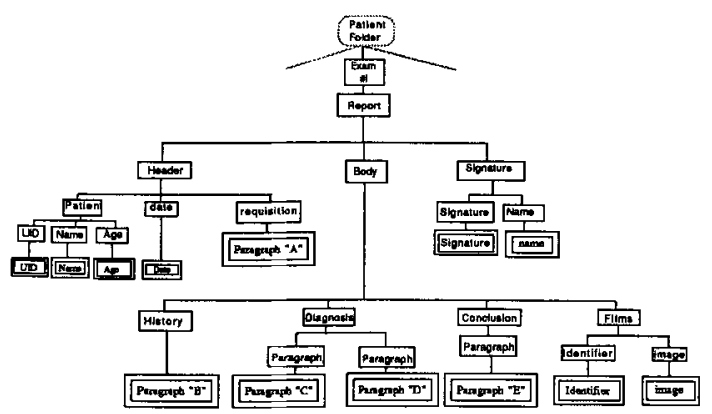

Fig 3. Specific logical structure of the static report example.

the presentation surface, eg, for imaging onto a sheet of paper; (3) frames that are used to define areas that constrain the layout of a page, eg, a column area, a header or footer area, or other particular areas such as those used on forms; and (4) blocks that are used for positioning particular content portions, eg, a paragraph, picture, or author's name. Figure 4 shows the hierarchical relationship between these components.

\section{Generic Structures}

The concept of object classes is used in the document architecture to define objects with common characteristics. The generic structure of a document describes characteristics common to a number of objects within a document. The generic logical structure is used to specify the rules applicable to the editing of a document as intended by the creator of the generic structure, achieved by defining the specific structures possible in a given document class and the object

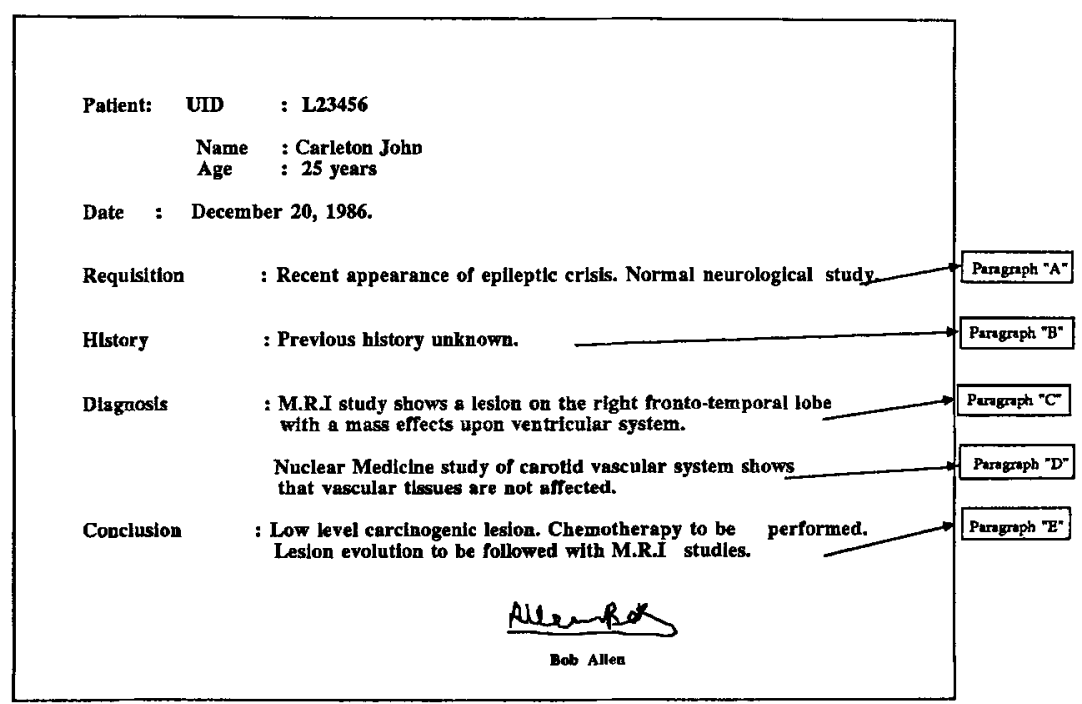

Fig 2. Example of a simple static report. 


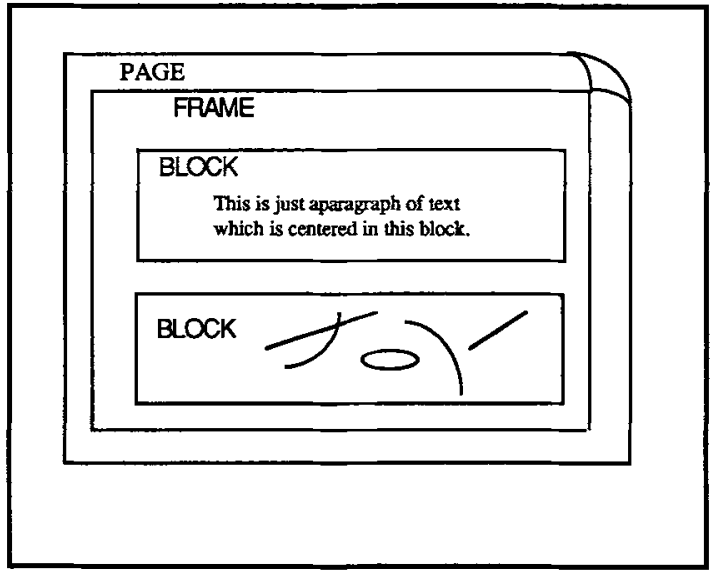

Fig 4. Hierarchy of pages, frames, and blocks.

classes possible in parts of a document. The generic layout structure specifies the rules for laying out the content of a document during editing or printing, thus supporting a "what you see is what you get" style of editing.

\section{Specific Structures}

The specific structures are defined as the structures of the document generated during the editing process according to the rules defined in the generic structures. A specific structure is an instance of the generic structure. The specific structures of a document consist of the specific logical structure and the specific layout structure. During the editing process of a document, the generic logical structure is used to control the document creation. The structure of the document generated within this process is termed specific logical structure. When the document has to be printed or displayed on the screen, the generic layout structure is used to generate the specific layout structure which is, in turn, involved in the image process that constitutes the last step before the display or the printing of the document.

\section{CONTENT PORTIONS}

The content of a document is divided into content portions to allow the document architecture to address each content portion as a unit. Within the structure of the document, content portions are associated with the basic objects that are the lowest-level components of a document. Content portions may include the type text, graphics, voice, and images. Each of these con- tent types is associated with a content architecture. Each content architecture specifies the rules applicable to the structure and the presentation of the particular content type. Different contents may be defined for a given document, depending on the user's application needs. The use of content architecture standards, wherever possible, is suitable for interchange purpose. The current version of ODA (ISO 8613) ${ }^{1}$ identifies three content types: characters, raster graphic (photographic images), and geometric graphics. Geometric graphics and raster graphics are based on ISO 8632-Computer Graphic Metafile (CGM) and International Telegraph and Telephone Consultative Committee (CCITT) Recommendation T.6, respectively. Content architectures for voice content have not yet been addressed by standards committees.

\section{DOCUMENT AS AN ENTITY}

A document considered as an entity of information to be exchanged and manipulated has certain properties that describe the document as a whole. These properties include document title, author, version number, abstract, keywords, etc. This set of properties is termed the Document Profile. For instance, a document can be indexed and stored in a database according to some properties included in the document profile so that it can be retrieved later by using the same indexing criteria. Also included in the document profile is some particular information concerning the document structures themselves, such as logical, layout, and generic structures. When a document is to be sent from one system to another, the document profile may first be exchanged before starting the transfer of the whole document, in order to make sure that the remote system has all the capabilities necessary for the treatment of the corresponding document.

\section{APPLICATION TO THE MEDICAL DOMAIN}

The concepts described herein are mainly defined in the ODA standard. As we previously mentioned, ODA has been studied in the context of office applications. In the following sections, we describe how these concepts may apply to medical applications. The objective is to define an application profile that permits a complete representation of the main document classes that can be found in a typical medical environment. 
This application profile defines the ODA standard subsets that are applicable to the medical domain, and proposes additional features that are required but not supported by the current version of ODA. These features include the handling of voice, annotation, forms, simultaneous treatment of objects in the structures, (see below) and medical images (architecture and compression/expansion algorithms). This study, however, is limited in the first stage to the radiology department. In the following sections we focus more specifically on the diagnosis report, treatments in terms of organization, architecture, generation, and playback.

\section{Diagnostic Report Organization}

In order to apply the ODA model to the medical report, we need to analyze and understand the kind of information we may find in the report, how these types of information are organized in terms of logical structure, and the possible links between the report's components and the films. Traditional diagnostic reports may contain such information as text, graphics, and images. This information may be organized as follows: a header that contains information related to the patient's examination such as patient name, exam's title and date, the requisition order, etc; a body that may contain the short patient history, diagnosis descriptions, and the conclusions or opinions; and a signature that ratifies the content of the report. These constituent elements are not always explicitly indicated in some existing manually generated reports. However, for generalization, we assume that all the reports are composed of the above main components and, because of these common characteristics, they pertain to the same report class.

The types of data that can be found in the paper reports may vary from one report to a nother. We consider data to be a combination of text, graphics (drawing, curves, etc), and image (x-rays) types. A voice type should be added when the report is electronically generated. Voice utilization is an important feature in the reportediting process. It could be used for a complete voice report generation or for annotations.

In the report-generation process radiologists read radiographic images of patients and report their finding to the referring physicians. The physician reads the report content to get the radiologist's findings. Once the report is generated, images used in the generation process become less important than the report itself. However, to answer the requisition statement it is often necessary to inspect previous examinations. In this case images may be read together with the report by the radiologist. There are different possible scenarios for generating and reading diagnostic reports. The radiologist reads all the images and then reports the findings. In this scenario there is no direct link between an image and its description part in the report. The report may be read by the physician without reading the images. In the case where images related to a report must be read they will be retrieved separately from the corresponding report. The second scenario mixes images and their description parts into the report; thus each image is linked to the corresponding description. Furthermore, different parts of an image could be described and linked with their descriptions in the report. Reading a report generated according to this scenario is performed in an animated way (playback). The goal of the document architecture is to propose means by which the above report generation and reading scenarios could be represented and described with respect to the logical structure and the synchronization requirements between images and their descriptions.

\section{Diagnostic Report Architecture}

Construction rules. The principal concepts used to express the structures of diagnosis reports are the notions of abstract objects, hierarchical links between objects, ordered and unordered objects, and shared components. All these concepts are represented in a tree structure allowing a diagnosis report to be fully described graphically.

We now describe the main rules that should apply to the report generation from the logical point of view. These rules are derived for the previously described traditional diagnostic report. They will be used in the construction of the generic logical structure. (1) There should be one report per examination (different examinations may exist for a given patient). All information related to that examination is integrated in the report; an example is the requisition form and the films used in the report generation. (2) The report is mainly composed of three parts: a 
header; a body; and signature with the name of the report editor. (3) The header is composed of a patient name, unique identifier and age, a date, and the requisition form that may be composed of one or more paragraphs. (4) The body is composed of the patient history of one or more paragraphs, the diagnostic findings, and a conclusion composed of one or more paragraphs and/or figures.

These rules specify the report structures in terms of composite and basic objects and the hierarchical relationships between these objects. In addition to these rules, we should indicate the order in which the components are to be examined and treated, which is necessary when the report is to be displayed on the screen or printed on paper. Two examination orders are to be considered in a hierarchical structure: sequential and simultaneous. The sequential order indicates that a set of objects belonging to a given node in the tree should be treated from left to right, one after the other. The simultaneous order indicates that a set of subordinate objects belonging to a node are treated at the same time from left to right. Order expresses the semantic relationships between the components of the report. For instance, paragraphs of the report should be read sequentially in order to capture their semantic meaning, and an image must be displayed together with its corresponding descriptive paragraphs.

Two classes of reports are to be defined in terms of structures: static and dynamic reports. In the static report there is no direct link between content and the images used in the report generation process. This class of reports is similar to the current methodology used in the radiology department, where radiologists report findings by analyzing radiographs and dictating reports, which are transcribed into hard-copy reports. The generated report is made available to the physician who can access it without having to look at the associated images. Dynamic report is a new method for generating and consulting diagnosis reports. The consultation of the report by the physician is performed in an animated fashion. The term animation is used to express the simultaneous treatment (eg, display) of the image and the associated descriptions.
Generic logical structure of the report. The generic logical structure of the report specifies the set of rules by which the report has to be constructed. The generic structure is very similar to the concept of a class in object oriented programming languages and the generic structure of a report is referred to as its report class. The generic structure of the static report class is defined in Fig 5. Figure 6 describes the generic structure for the dynamic report class. However, the two structures are given as examples of possible report organizations; other structures for different diagnostic report organizations could be defined by using the same construction method.

In Fig 5 the generic structure defines a report as a sequence of a header, body and signature, and name. The header in turn is composed of patient unique identifier, name, age, date of the report, and the requisition form constituted of one or more paragraphs. The body part of the report is a sequence of a patient history comprising at least one paragraph, a diagnosis that may contain either one or more paragraphs and/or figures, a conclusion formed of paragraphs, and a set of ordered films composed of an identifier and the associated image. The use of figures and patient history in the structure are assumed to be optional.

In Fig 6 the generic structure represents a dynamic report. The difference between this structure and the one described in Fig 5 resides in the diagnosis section of the body part. This section contains the film and the corresponding description subsections. The film section is a sequence of identifier and associated images. The description subsections may report findings re-

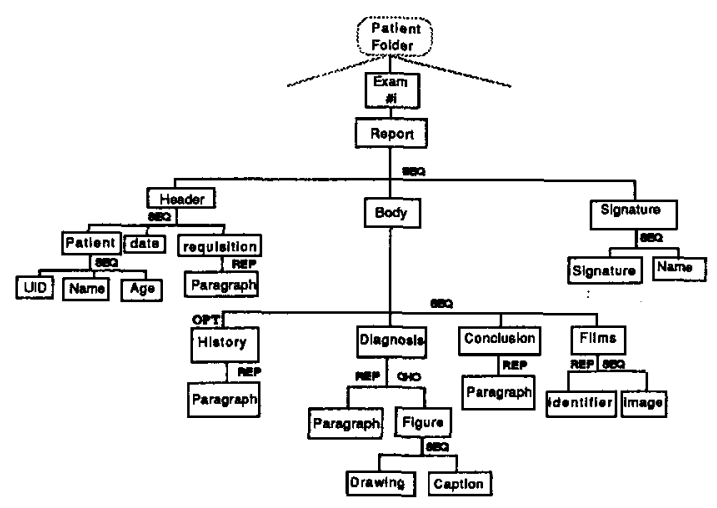

Fig 5. Generic logical structure of the static report. 


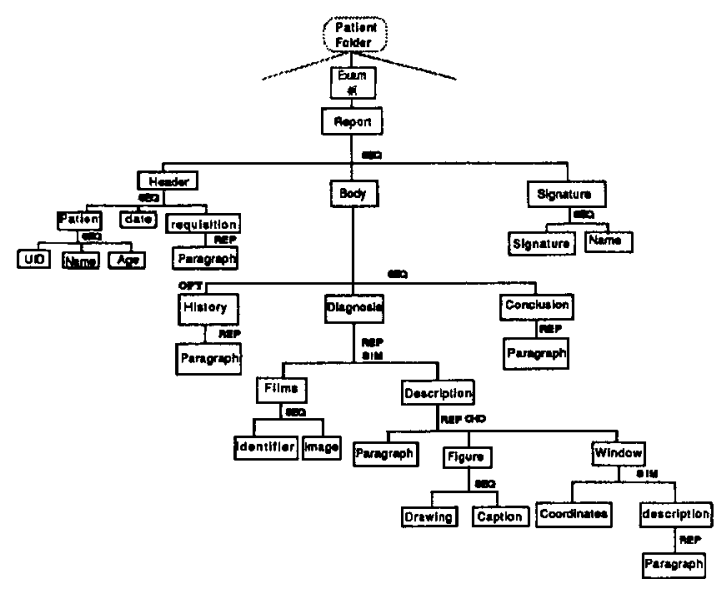

Fig 6. Generic logical structure of the dynamic report.

lated to the image as a whole. In this case, paragraphs and figure units are used for this purpose. If more detailed description is necessary, the window section could be used to indicate the position to be highlighted (the position may be located, in the image, by means of graphic elements such as a circle for example) and to identify the findings associated with it.

The specific logical structure of the report. The specific structure of the report is derived from the generic structure, it represents an instance of the report class represented by the generic structures described above. Figure 2 gives an example of a static report as it may be printed or displayed on the screen. The corresponding specific logical structure generated, with respect to the generic logical structure, is shown in Fig 3. Content portions are located at the lowest level components of the tree; they are only of text type in this example. However, other content types may be specified. Content architecture defined in the current version of ODA may be used for text, geometric, and raster graphics. A specific content architecture is necessary for radiographic images that defines the image coding principles and the associated compression algorithm.

\section{CONCLUSION}

A document architecture for medical application using the ODA concepts with some extensions and adaptations has been briefly described in this article. Document architecture design is a part of the research being conducted at the University of Ottawa toward the design of a multimedia distributed database system for the MUSIC project. ${ }^{4}$ As we have mentioned previously, document architecture is a key factor in the design of the PACS that needs to be clearly defined in order to understand and organize information that has to be processed by different and geographically distributed applications such as editor, database, image processing, and coding, etc. European Computer Manufacturers Association (ECMA) and CCITT are among other organizations, beside ISO, that have produced respectively ECMA- $101^{5}$ and recommendations on T. $73^{6}$ and T.400. ${ }^{7}$ In the medical environment, American College of RadiologyNational Electrical Manufacturers Association ${ }^{8}$ standard committee is the most qualified organization to conduct work items on the multimedia document architecture. It may be based on the existing standards, with additional enhancements, in order to satisfy the functionality required of future interworking PACS.

\section{REFERENCES}

1. ISO, Information Processing-Text and Office Systems. Office document architecture (ODA) and interchange Format (ODIF). Part one to eight. International Standard 8613, (March 1988)

2. Nosil J, Justice C: A prototype multi-modality picture and communication system at Victoria General Hospital. Medical Imaging II: Image Data Management and Display 914:1363-1378, 1988 (part B)

3. Hedge SS, et al: AT\&T PACS Architecture. Picture Archiving and Communication Systems (PACS IV) for Medical Applications. SPIE, 1986, pp 618-625

4. Georganas ND, et al: Multimedia system for integrated communications (the project MUSIC). Research report.
Electrical Engineering. Ontario, Canada, University of Ottawa, August 1987, p 56

5. ECMA. ECMA-101: Office Document Architecture, 1985

6. CCITT. Recommendation T.73-Document Interchange Protocol for Telematic Services. Geneva, 1984

7. CCITT Draft Recommendation T4xa-Document transfer Architecture-General principles. Document Architecture, Application Rules. Geneva, 1986

8. American National Standard Institute: ACR-NEMA Standards Publications No. 300-1985. Digital Imaging and Communications, NEMA, 1985 